\title{
Midazolam for status epilepticus
}

\section{SUMMARY}

Midazolam is now the first-line treatment for status epilepticus in children.

The drug can be given via several different routes. Transmucosal administration is safe, effective, easier to use and more socially acceptable than rectal diazepam.

The aim is to stop convulsive seizures that have lasted longer than five minutes as soon as possible.

Transmucosal pre-hospital administration by carers is recommended for patients with a predilection for prolonged seizures, or those with limited access to emergency services. This reduces the time to treat and improves outcomes.

General practitioners can obtain midazolam through the Prescriber Bag Drug Supplies section of the Pharmaceutical Benefits Scheme. For pre-hospital use, $5 \mathrm{mg}$ in $1 \mathrm{~mL}$ plastic ampoules is recommended.

\section{Introduction}

Midazolam is a benzodiazepine which has been used in the treatment of status epilepticus since the early 1990s. Midazolam has replaced diazepam as the preferred first-line drug for acute management of seizures in infants and children. It is not currently registered for this indication in Australia, but in 2015 midazolam was made available for emergency use by GPs through the Pharmaceutical Benefits Scheme and is widely used off label for the treatment of seizures. Midazolam may also be administered by parents and carers at home and school, by ambulance officers and in the emergency department.

\section{Treating status epilepticus}

Most convulsive seizures do not last longer than five minutes and resolve without medical intervention. Any convulsive seizure lasting longer than five minutes should be treated as there is an increased risk of neuronal compromise following prolonged seizures.

Drugs, such as midazolam, are used to abort ongoing seizures and thereby avoid the complications of prolonged status epilepticus. The drugs resolve the majority of emergency presentations and they work best if given soon after the seizure has exceeded five minutes. Drug treatment becomes less effective if the seizure lasts longer than 15 minutes. ${ }^{2}$ Repeat doses may be effective but increase the risk of complications and sometimes inappropriately delay administration of second-line therapy.

\section{First-line drugs}

For many years, intravenous or rectal diazepam was the first-choice drug for stopping status epilepticus. It is effective but can be difficult to use, and rectal administration was less socially acceptable outside hospital.

Midazolam is a water-soluble benzodiazepine which can be given intramuscularly, intravenously or transmucosally. It was incorporated into a guideline for the management of seizures published by the New South Wales (NSW) Ministry of Health in 2009. ${ }^{3}$ The 2016 version of this guideline says that midazolam is the drug of first choice when intravenous access has not been obtained. ${ }^{4}$ Administering midazolam outside hospital reduces the time-to-treat period and improves outcomes. ${ }^{5}$ Midazolam can also be used to terminate clusters of brief convulsive seizures and manage bouts of nonconvulsive status epilepticus.

\section{Second-line drugs}

Established second-line drugs include phenytoin, phenobarbitone and levetiracetam. These are given after transfer to an emergency facility if first-line drugs fail to stop the seizures.

\section{Emergency use of midazolam}

The aims of giving midazolam are to avoid progression to, and the complications of, convulsive status epilepticus. Early treatment improves outcomes and avoids complications.

Plastic ampoules of $5 \mathrm{mg}$ in $1 \mathrm{~mL}$ (containing approximately 18 drops) are easier to use than the

\section{Rob Smith}

Paediatric neurologist

Conjoint senior lecturer ${ }^{2}$

\section{Janis Brown}

Clinical nurse consultant epilepsy

' John Hunter Children's

Hospital

Newcastle

2 Faculty of Health and Medicine

University of Newcastle New South Wales

\section{Keywords}

Doctor's Bag, emergency drugs, midazolam, Pharmaceutical Benefits Scheme, status epilepticus

Aust Prescr 2017:40:23-5 http://dx.doi.org/10.18773/ austprescr.2017.005 


\section{Fig. 1 Technique for administering buccal midazolam}

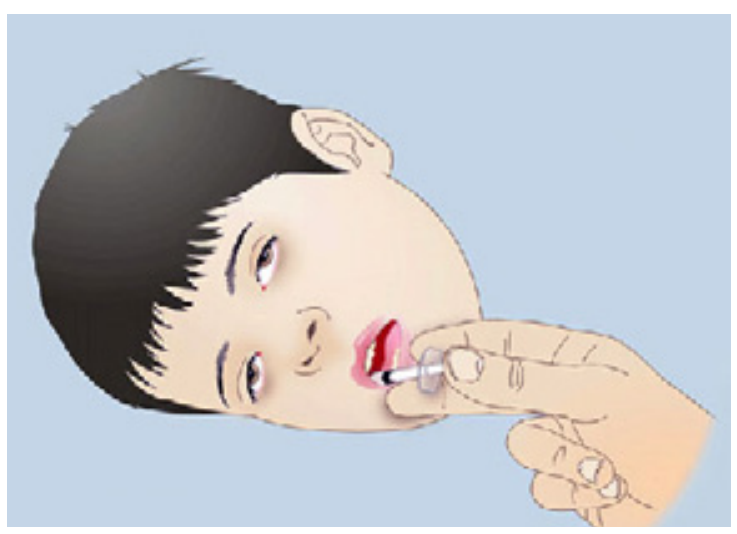

Reproduced with permission. The Royal Children's Hospital, Melbourne, Australia; Kids Health Info; www.rch.org.au/kidsinfo

glass ampoules. They should be protected from light, kept in their foil wrapper until required and stored at $15-25^{\circ} \mathrm{C}$.

Current guidelines recommend an initial buccal or intranasal dose of $0.3 \mathrm{mg} / \mathrm{kg}$ to a maximum of $10 \mathrm{mg} .{ }^{4}$ Each drop of the $5 \mathrm{mg} / \mathrm{mL}$ solution contains approximately $0.3 \mathrm{mg}$ midazolam. Absorption takes approximately 1-3 minutes and midazolam can take up to 10 minutes to abort the seizure. The dose can be repeated after five minutes if seizures persist. The techniques for administration are described on the websites of the Royal Children's Hospital Melbourne $^{6,7}$ and the Paediatric Epilepsy Network $\mathrm{NSW}^{8}$ (see Figs 1 and 2).

Ambulance officers and paramedics in NSW use midazolam in single or repeated doses of $0.15 \mathrm{mg} / \mathrm{kg}$ intramuscularly or intravenously for children in convulsive status epilepticus. They can give adults cumulative $2.5-5 \mathrm{mg}$ intravenous doses of up to $15 \mathrm{mg}$ in total. ${ }^{9}$ Emergency departments follow the relevant state guidelines for children and adults with midazolam as the first-choice therapy. ${ }^{3,10}$

\section{Administration by carers}

When used in the community midazolam can reduce hospital admissions for children with complex epilepsy." Providing a supply of midazolam to a parent or carer can be considered for children (and dependent adults) who have convulsive seizures which frequently last more than five minutes.

Parents and carers can be anxious about giving midazolam so training is needed. Education is available from specialist epilepsy nurses and can

\section{Fig. 2 Technique for administering} intranasal midazolam

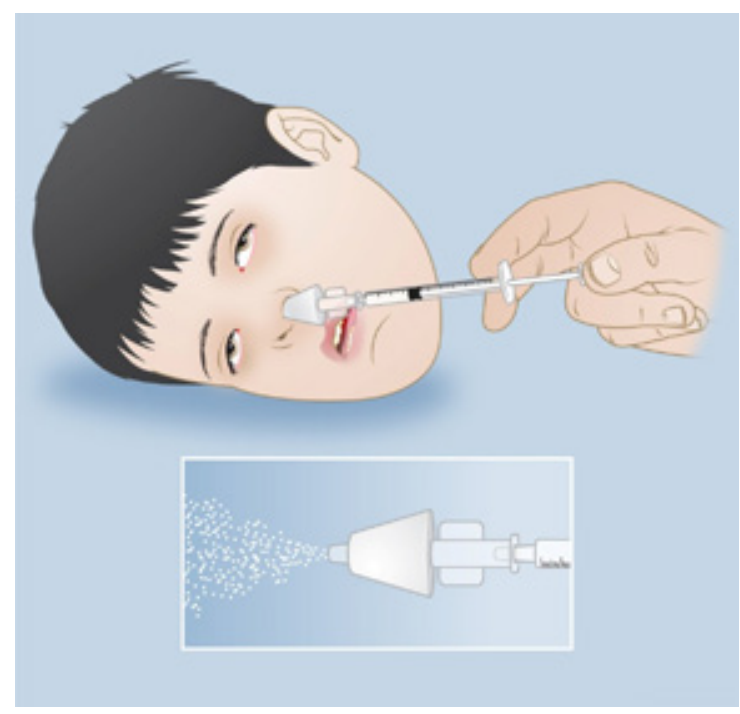

Reproduced with permission. The Royal Children's Hospital, Melbourne, Australia; Kids Health Info; www.rch.org.au/kidsinfo

be arranged through patient support organisations such as Epilepsy Action (www.epilepsy.org.au) and Epilepsy Australia (www.epilepsyaustralia.net). Prepare clear concise directions for administration. A suitable administration template can be found on the Paediatric Epilepsy Network NSW website. ${ }^{8}$

\section{Safety and tolerability}

The common adverse effects of transmucosal midazolam are sedation, ataxia, irritability or euphoria, and mild respiratory depression. Intranasal administration may also produce local irritation, stinging, sometimes with eyes watering and a runny nose.

Avoid using the intranasal route in children with any awareness during seizures because of the irritation. We recommend using a reusable mucosal atomisation device for intranasal administration, delivering half the dose into each nostril. Mucosal atomisation devices may be available through hospitals or the ambulance service and can be purchased privately.

Major complications of treatment are unlikely when the guidelines for midazolam are followed. ${ }^{12}$ With a single dose, respiratory depression is rare. Cumulative subsequent doses are much safer if given where expert airway support is available.

When midazolam is provided for use by carers, we recommend nominating only one prescriber 
and one dispenser for each child in order to avoid confusion. This is most often the treating neurologist and the hospital pharmacy because of the restricted availability of the plastic ampoules. Midazolam is available in a range of concentrations and volumes so we restrict prescription to $5 \mathrm{mg}$ in $1 \mathrm{~mL}$ plastic ampoules, wherever possible, to avoid dosing errors. Having a single prescriber and dispenser also reduces the potential for abuse and stockpiling. In a review of four years of pre-hospital use we found only two out of 197 families with circumstantial evidence of carers abusing the drug. ${ }^{13}$

Parents used to using plastic ampoules may struggle without extra instruction if glass ampoules are dispensed. It is always best to review parental understanding and techniques periodically. Frequent problems include:

- loss of dose from 'salivary washout' in dribbly children

- dose swallowing in partially aware children
- blocked noses

- confusion between ' $m L$ ' and 'mg' and the risk of accidental overdosing

- failure to adjust doses for growing children.

\section{Conclusion}

Midazolam is a benzodiazepine which is now the recommended first-line drug for treating convulsive status epilepticus. When used according to guidelines for infants, children and adults it appears safe and effective.

The use of midazolam by carers in the community is a widely accepted but off-label practice. It must be carefully planned, supervised and controlled. $<$

Conflict of interest: none declared

\section{REFERENCES}

1. Trinka E, Cock H, Hesdorffer D, Rossetti AO, Scheffer IE, Shinnar S, et al. A definition and classification of status epilepticus--Report of the ILAE Task Force on Classification of Status Epilepticus. Epilepsia 2015;56:1515-23. http://dx.doi.org/10.1111/epi.13121

2. Shorvon S. Handbook of epilepsy treatment. 3rd ed. Oxford: Wiley-Blackwell; 2010.

3. NSW Government Health. Children and infants with seizures - acute management. 16 October 2009. http://www0.health.nsw.gov.au/policies/ pd/2009/PD2009_065.html [cited 2017 Jan 9]

4. NSW Government Health. Infants and children: acute management of seizures. 4 February 2016. www0.health.nsw.gov.au/policies/pd/2009/ PD2009_065.html [cited 2017 Jan 9]

5. Brigo F, Nardone R, Tezzon F, Trinka E. Nonintravenous midazolam versus intravenous or rectal diazepam for the treatment of early status epilepticus: A systematic review with meta-analysis. Epilepsy Behav 2015;49:325-36. http://dx.doi.org/10.1016/j.yebeh.2015.02.030

6. The Royal Children's Hospital Melbourne. Kids health info. Buccal midazolam. Updated April 2014. http://www.rch.org.au/kidsinfo/fact sheets/ Buccal_midazolam [cited 2017 Jan 9]

7. The Royal Children's Hospital Melbourne. Kids health info. Intranasal midazolam. 2014. http://www.rch.org.au/kidsinfo/fact sheets/Intranasal Midazolam [cited 2017 Jan 9]
8. Paediatric Epilepsy Network NSW. Children's epilepsy resources for clinicians. Epilepsy management documents. 2016. http://www.pennsw.com.au/ clinician-resources/epilepsy-resources.html [cited 2017 Jan 9]

9. Ambulance Service of New South Wales. Protocols and pharmacology January 2011. http://www.ambo.com.au/download/protocol_2011.pdf [cited 2017 Jan 9]

10. NSW Government Health. Rural adult emergency clinical guidelines. 4th ed. 10 May 2016. http://www0.health.nsw.gov.au/policies/gl/2016/GL2016_012. html [cited 2017 Jan 9]

11. Klimach VJ; Epic Clinical Network. The community use of rescue medication for prolonged epileptic seizures in children. Seizure 2009;18:343-6. http://dx.doi.org/10.1016/j.seizure.2008.12.002

12. Glauser T, Shinnar S, Gloss D, Alldredge B, Arya R, Bainbridge J, et al. Evidence-based guideline: treatment of convulsive status epilepticus in children and adults: report of the Guideline Committee of the American Epilepsy Society. Epilepsy Curr 2016;16:48-61. http://dx.doi.org/10.5698/ 1535-7597-16.1.48

13. Brown J, Subramanian G, Miteff C, Fardell B, Smith R. Trends in prescribing home midazolam for prolonged seizures in children in suburban and rural northern NSW: a 4 year retrospective analysis. Poster presented at the 26th Annual Scientific Meeting of the Epilepsy Society of Australia; 2012; Hobart.

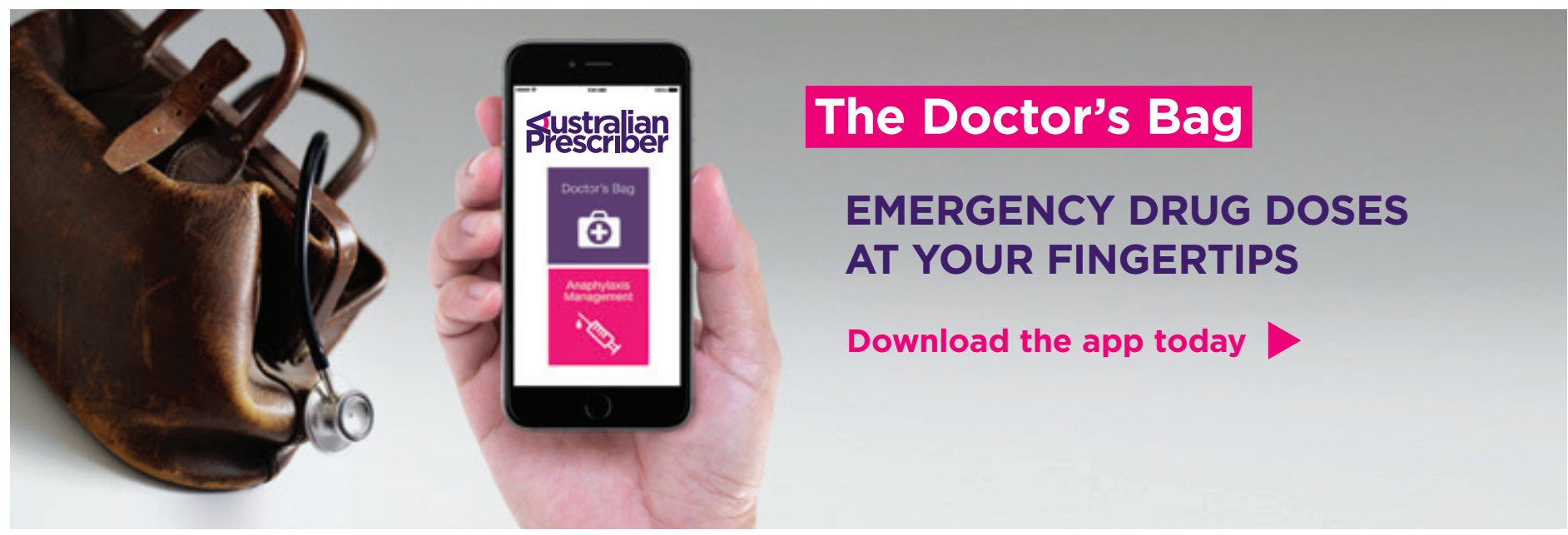

